\title{
Retraction of: Ozayar et al., DOI: 10.1089/bari.2015.0043
}

The Editor of Bariatric Surgical Practice and Patient Care is officially retracting the article published online entitled, "The Effect of Bougie Size on the Incidence of Postoperative Sore Throat in Bariatric Surgery," by Ozayar E. et al. DOI: 10.1089/ bari.2015.0043.

This article was found to be an earlier version of an already published article that had been finalized under the DOI: 10.1089/ bari.2015.0035.

The Publishers deeply regret this error. 